\title{
AN OPTIMAL DIVIDENDS PROBLEM WITH A TERMINAL VALUE FOR SPECTRALLY NEGATIVE LÉVY PROCESSES WITH A COMPLETELY MONOTONE JUMP DENSITY
}

\author{
R. L. LOEFFEN, ${ }^{*}$ Austrian Academy of Sciences
}

\begin{abstract}
We consider a modified version of the classical optimal dividends problem of de Finetti in which the objective function is altered by adding in an extra term which takes account of the ruin time of the risk process, the latter being modeled by a spectrally negative Lévy process. We show that, with the exception of a small class, a barrier strategy forms an optimal strategy under the condition that the Lévy measure has a completely monotone density. As a prerequisite for the proof, we show that, under the aforementioned condition on the Lévy measure, the $q$-scale function of the spectrally negative Lévy process has a derivative which is strictly log-convex.
\end{abstract}

Keywords: Lévy process; stochastic control; dividend problem; scale function; complete monotonicity

2000 Mathematics Subject Classification: Primary 60J99

Secondary 93E20; 60G51

\section{Introduction}

In this paper we consider the classical de Finetti's optimal dividends problem, but with an extra component regarding the ruin time added to the objective function. Within this problem we assume that the underlying dynamics of the risk process are described by a spectrally negative Lévy process, which is now widely accepted and used as a replacement for the classical Cramér-Lundberg process (cf. [1], [3], [8], [9], [11], [14], [16], [19], [20], and [23]). Recall that a Cramér-Lundberg risk process $\left\{X_{t}: t \geq 0\right\}$ corresponds to

$$
X_{t}=x+c t-\sum_{i=1}^{N_{t}} C_{i},
$$

where $x>0$ denotes the initial surplus, the claims $C_{1}, C_{2}, \ldots$ are independent and identically distributed positive random variables with expected value $\mu, c>0$ represents the premium rate, and $N=\left\{N_{t}: t \geq 0\right\}$ is an independent Poisson process with arrival rate $\lambda$. Traditionally, it is assumed in the Cramér-Lundberg model that the net profit condition $c>\lambda \mu$ holds, or, equivalently, that $X$ drifts to $\infty$. In this paper, $X$ will be a general spectrally negative Lévy process and the condition that $X$ drifts to $\infty$ will not be assumed.

We will now state the control problem considered in this paper. As mentioned before, $X=\left\{X_{t}: t \geq 0\right\}$ is a spectrally negative Lévy process which is defined on a filtered probability space $\left(\Omega, \mathcal{F}, \mathbb{F}=\left\{\mathcal{F}_{t}: t \geq 0\right\}, \mathrm{P}\right)$ satisfying the usual conditions. Within the definition of a

Received 9 June 2008; revision received 25 November 2008.

* Postal address: Radon Institute for Computational and Applied Mathematics, Austrian Academy of Sciences, Altenbergerstrasse 69, A-4040 Linz, Austria. Email address: ronnie.loeffen@oeaw.ac.at 
spectrally negative Lévy process, it is implicitly assumed that $X$ does not have monotone paths. We denote by $\left\{\mathrm{P}_{x}, x \in \mathbb{R}\right\}$ the family of probability measures corresponding to a translation of $X$ such that $X_{0}=x$, where we write $\mathrm{P}=\mathrm{P}_{0}$. Furthermore, $\mathrm{E}_{x}$ denotes the expectation with respect to $\mathrm{P}_{X}$, with $\mathrm{E}$ being used in the obvious way. The Lévy triplet of $X$ is given by $(\gamma, \sigma, \nu)$, where $\gamma \in \mathbb{R}, \sigma \geq 0$, and $v$ is a measure on $(0, \infty)$ satisfying

$$
\int_{(0, \infty)}\left(1 \wedge x^{2}\right) v(\mathrm{~d} x)<\infty
$$

Note that even though $X$ has only negative jumps, for convenience, we choose the Lévy measure to have mass only on the positive instead of the negative half line. The Laplace exponent of $X$ is given by

$$
\psi(\theta)=\log \left(\mathrm{E}\left(\exp \left(\theta X_{1}\right)\right)\right)=\gamma \theta+\frac{1}{2} \sigma^{2} \theta^{2}-\int_{(0, \infty)}\left(1-\mathrm{e}^{-\theta x}-\theta x \mathbf{1}_{\{0<x<1\}}\right) v(\mathrm{~d} x),
$$

and is well defined for $\theta \geq 0$. Note that the Cramér-Lundberg process corresponds to the case in which $\sigma=0, v(\mathrm{~d} x)=\lambda F(\mathrm{~d} x)$, where $F$ is the law of $C_{1}$, and $\gamma=c-\int_{(0,1)} x v(\mathrm{~d} x)$. The process $X$ will represent the risk/surplus process of an insurance company before dividends are deducted.

We denote a dividend or control strategy by $\pi$, where $\pi=\left\{L_{t}^{\pi}: t \geq 0\right\}$ is a nondecreasing, left-continuous, $\mathbb{F}$-adapted process which starts at 0 . The random variable $L_{t}^{\pi}$ will represent the cumulative dividends the company has paid out until time $t$ under the control $\pi$. We define the controlled (net) risk process $U^{\pi}=\left\{U_{t}^{\pi}: t \geq 0\right\}$ by $U_{t}^{\pi}=X_{t}-L_{t}^{\pi}$. Let $\sigma^{\pi}=\inf \{t>$ $\left.0: U_{t}^{\pi}<0\right\}$ be the ruin time, and define the value function of a dividend strategy $\pi$ by

$$
v_{\pi}(x)=\mathrm{E}_{x}\left(\int_{0}^{\sigma^{\pi}} \mathrm{e}^{-q t} \mathrm{~d} L_{t}^{\pi}+S \exp \left(-q \sigma^{\pi}\right)\right)
$$

where $q>0$ is the discount rate and $S \in \mathbb{R}$ is the terminal value. By definition, it follows that $v_{\pi}(x)=S$ for $x<0$. A strategy $\pi$ is called admissible if ruin does not occur due to a lump sum dividend payment, i.e. $L_{t+}^{\pi}-L_{t}^{\pi} \leq U_{t}^{\pi} \vee 0$ for $t \leq \sigma^{\pi}$. Let $\Pi$ be the set of all admissible dividend policies. The control problem consists of finding the optimal value function $v_{*}$ given by

$$
v_{*}(x)=\sup _{\pi \in \Pi} v_{\pi}(x)
$$

and an optimal strategy $\pi_{*} \in \Pi$ such that

$$
v_{\pi_{*}}(x)=v_{*}(x) \text { for all } x \geq 0 .
$$

When $S=0$, the above optimal control problem transforms, albeit within the more general framework of a spectrally negative Lévy risk process, to the original optimal dividends problem introduced firstly in a discrete-time setting by de Finetti [7] and later studied in, amongst others, [3], [4], [12], [19], and [20]. The general case when $S \in \mathbb{R}$ that we consider here is not new. Thonhauser and Albrecher [26] studied in the Cramér-Lundberg setting the case in which $S<0$. In this case the extra term added to the value function penalizes early ruin and so this model can be used if, besides the value of the dividend payments, we also want to take into consideration the lifetime of the risk process. The parameter $S$ can then be used to find the desired 'balance' between optimizing the value of the dividends and maximizing the ruin time. 
When $S>0$, the model can be used if the company, when it becomes bankrupt, has a salvage value equaling $S$ which is distributed to the same beneficiaries as the dividends are; see also the discussion in [22, Section 3]. In a Brownian motion/diffusion setting, this control problem has been studied in [5] and [24].

We will now introduce two types of dividend strategies and state our main theorem. We denote by $\pi_{a}=\left\{L_{t}^{a}: t \geq 0\right\}$ the barrier strategy at level $a \geq 0$ with corresponding value function $v_{a}$ and ruin time $\sigma^{a}$. This strategy is defined by $L_{0}^{a}=0$ and

$$
L_{t}^{a}=\left(\sup _{0 \leq s<t} X_{s}-a\right) \vee 0 \text { for } t>0 .
$$

Note that $\pi_{a} \in \Pi$. So, if dividends are paid out according to a barrier strategy with the barrier placed at $a$, then the corresponding controlled risk process will be a spectrally negative Lévy process reflected in $a$.

We further introduce the take-the-money-and-run strategy $\pi_{\text {run }}=\left\{L_{t}^{\text {run }}: t \geq 0\right\}$, which is the strategy where all of the surplus of the company is directly paid out and immediately thereafter ruin is forced (note that ruin is defined as the state when the controlled risk process is strictly below 0). The value of this strategy is $v_{\text {run }}(x)=x+S$ for $x \geq 0$. In case $X$ is not a Cramér-Lundberg risk process, this strategy is the same as the barrier strategy with the barrier placed at 0 (i.e. almost surely (a.s.), $L_{t}^{0}=L_{t}^{\text {run }}$ for all $t \geq 0$ ). But, if $X$ is a CramérLundberg risk process then the barrier strategy at 0 does not imply immediate ruin; ruin occurs only after the first jump/claim which takes an exponentially distributed with parameter $v(0, \infty)$ amount of time. Therefore, the value of the latter strategy might be different than the value of the take-the-money-and-run strategy. In particular, for large terminal values, $v_{\text {run }}$ might be bigger than $v_{0}$ since it can be beneficial to become ruined as soon as possible. Note that in the Cramér-Lundberg case, ruin can be forced in an admissible way by paying out dividends at a rate which is larger than the premium rate immediately after taking out all the surplus.

Recall that an infinitely differentiable function $f:(0, \infty) \rightarrow[0, \infty)$ is completely monotone if its derivatives alternate in sign, i.e. $(-1)^{n} f^{(n)}(x) \geq 0$ for all $n=0,1,2, \ldots$ and all $x>0$. The main theorem of this paper reads as follows.

Theorem 1. Suppose that the Lévy measure of the spectrally negative Lévy process $X$ with Lévy triplet $(\gamma, \sigma, v)$ has a completely monotone density. Let $c=\gamma+\int_{0}^{1} x v(\mathrm{~d} x)$. Then the following statements hold.

(i) If $\sigma>0$, or $v(0, \infty)=\infty$, or $v(0, \infty)<\infty$ and $S \leq c / q$, then an optimal strategy for the control problem is formed by a barrier strategy.

(ii) If $\sigma=0, v(0, \infty)<\infty$, and $S>c / q$, then the take-the-money-and-run strategy is an optimal strategy for the control problem.

Note that the parameter $c$ is strictly positive since we assumed that the paths of $X$ are not monotone decreasing. For $X$ being equal to a Brownian motion with drift, this control problem has been solved in [5] and [24]. In the case when $X$ is a Cramér-Lundberg process with exponentially distributed claims, the control problem was solved by Gerber [12] for $S=0$ and by Thonhauser and Albrecher [26] for $S<0$ (note that Thonhauser and Albrecher [26, Lemma 10] distinguished between the cases of the optimal strategy being a barrier strategy where the barrier is placed at 0 and where the barrier is placed at a strictly positive level, whereas we in Theorem 1 distinguish between the cases where a barrier strategy is optimal and where the take-the-money-and-run strategy is optimal). Note that both cases are examples 
for which the Lévy measure has a completely monotone density and, thus, are contained in Theorem 1. Some other examples of spectrally negative Lévy processes which have a Lévy measure with a completely monotone density can be found in [20].

Building on the work of Avram et al. [3], Loeffen [20] proved Theorem 1 for $S=0$. In particular, it was shown that optimality of the barrier strategy depends on the shape of the so-called scale function of a spectrally negative Lévy process. To be more specific, the $q$-scale function of $X, W^{(q)}: \mathbb{R} \rightarrow[0, \infty)$, where $q \geq 0$, is the unique function such that $W^{(q)}(x)=0$ for $x<0$, and on $[0, \infty)$ is a strictly increasing and continuous function characterized by its Laplace transform which is given by

$$
\int_{0}^{\infty} \mathrm{e}^{-\theta x} W^{(q)}(x) \mathrm{d} x=\frac{1}{\psi(\theta)-q} \text { for } \theta>\Phi(q),
$$

where $\Phi(q)=\sup \{\theta \geq 0: \psi(\theta)=q\}$ is the right inverse of $\psi$. Loeffen [20] showed that when $W^{(q)}$ is sufficiently smooth and $W^{(q) \prime}$ is increasing on $\left(a^{*}, \infty\right)$, where $a^{*}$ is the largest point where $W^{(q) \prime}$ attains its global minimum, then the barrier strategy at $a^{*}$ is optimal for the control problem (in the $S=0$ case). Here $W^{(q)}$ being sufficiently smooth means that $W^{(q)}$ is once or twice continuously differentiable when $X$ is of bounded or, respectively, unbounded variation. It was then shown in [20] that when $X$ has a Lévy measure which has a completely monotone density, these conditions on the scale function are satisfied and, in particular, that $W^{(q) \prime}$ is strictly convex on $(0, \infty)$. Shortly thereafter, Kyprianou et al. [19] showed that $W^{(q) \prime}$ is strictly convex on $\left(a^{*}, \infty\right)$ (but not necessarily on $(0, \infty)$ (see [19, Section 3])) under the weaker condition that the Lévy measure has a density which is log-convex. Though the scale function in that case is not necessarily sufficiently smooth, Kyprianou et al. [19] were able to circumvent this problem and proved that the barrier strategy at $a^{*}$ is still optimal when the Lévy measure has a log-convex density. Note that without a condition on the Lévy measure the barrier strategy is not optimal in general. Indeed, Azcue and Muler [4] have given an example for which no barrier strategy is optimal.

The proof of Theorem 1 in the case when $S \neq 0$ relies on the assumption that $W^{(q) \prime}$ is strictly log-convex on $(0, \infty)$. Though in [20] it was only shown, under the complete monotonicity assumption on the Lévy measure, that $W^{(q) \prime}$ is strictly convex on $(0, \infty)$, we will show in Section 2 that the stronger property of strict log-convexity actually holds in that case. Then in Section 3 the proof of Theorem 1 will be given.

\section{Scale functions}

Associated to the functions $\left\{W^{(q)}: q \geq 0\right\}$ mentioned in the previous section are the functions $Z^{(q)}: \mathbb{R} \rightarrow[1, \infty)$ defined by

$$
Z^{(q)}(x)=1+q \int_{0}^{x} W^{(q)}(y) \mathrm{d} y \quad \text { for } q \geq 0 .
$$

Together, the functions $W^{(q)}$ and $Z^{(q)}$ are collectively known as scale functions and predominantly appear in almost all fluctuation identities for spectrally negative Lévy processes. As an example, we mention the one-sided exit below problem for which

$$
\mathrm{E}_{x}\left(\mathrm{e}^{-q \tau_{0}^{-}} \mathbf{1}_{\left\{\tau_{0}^{-}<\infty\right\}}\right)=Z^{(q)}(x)-\frac{q}{\Phi(q)} W^{(q)}(x),
$$

where $\tau_{0}^{-}=\inf \left\{t>0: X_{t}<0\right\}$. 
We will now recall some properties of scale functions which we will need later on. When the Lévy process drifts to $\infty$ or, equivalently, $\psi^{\prime}(0+)>0$, the 0 -scale function $W^{(0)}$ (which will be denoted from now on by $W$ ) is bounded and has a $\operatorname{limit}_{x \rightarrow \infty} W(x)=1 / \psi^{\prime}(0+)$. Furthermore, for $q \geq 0$, there is the following relation between scale functions:

$$
W^{(q)}(x)=\mathrm{e}^{\Phi(q) x} W_{\Phi(q)}(x),
$$

where $W_{\Phi(q)}$ is the (0-)scale function of $X$ under the measure $\mathrm{P}^{\Phi(q)}$ defined by

$$
\left.\frac{\mathrm{dP}^{\Phi(q)}}{\mathrm{dP}}\right|_{\mathcal{F}_{t}}=\exp \left(\Phi(q) X_{t}-q t\right) .
$$

The process $X$ under the measure $\mathrm{P}^{\Phi(q)}$ is still a spectrally negative Lévy process and its Laplace exponent is given by $\psi_{\Phi(q)}(\theta)=\psi(\Phi(q)+\theta)-\psi(\Phi(q))$. When $q>0$, it is known that $\psi_{\Phi(q)}^{\prime}(0+)=\psi^{\prime}(\Phi(q))>0$.

From $[15,(8.18)]$ and the fact that $W$ is strictly positive on $(0, \infty)$, it follows that we can write, for $x, a>0$,

$$
\log (W(x))=\log (W(a))+\int_{a}^{x} g(t) \mathrm{d} t,
$$

where $g$ is a decreasing function and, hence, $\log (W(x))$ is concave on $(0, \infty)$ (see, e.g. [27, Theorem 1.13]). Recall here that a strictly positive function $f$ is said to be log-concave or $\log$-convex whenever $\log (f)$ is concave or, respectively, convex. From (2), it now follows that, for $q \geq 0, \log \left(W^{(q)}(x)\right)$ is concave on $(0, \infty)$ and, thus, $W^{(q)}$ is log-concave on $(0, \infty)$ for all $q \geq 0$.

The initial value of the scale function $W^{(q)}(0)$ is equal to $1 / c$, where $c$ is as in Theorem 1 . Note that if $X$ is of unbounded variation then $c=\infty$ and, thus, $W^{(q)}(0)=0$. The initial value of the derivative of the scale function is given by (see, e.g. [18])

$$
W^{(q) \prime}(0):=\lim _{x \downarrow 0} W^{(q) \prime}(x)= \begin{cases}\frac{2}{\sigma^{2}} & \text { when } \sigma>0, \\ \frac{v(0, \infty)+q}{c^{2}} & \text { when } \sigma=0 \text { and } v(0, \infty)<\infty, \\ \infty & \text { otherwise. }\end{cases}
$$

Despite the fact that the scale function is in general only implicitly known through its Laplace transform, there are plenty of examples of spectrally negative Lévy processes for which there exists closed-form expressions for their scale functions, although most of these examples only deal with the $q=0$ scale function. In case no explicit formula for the scale function exists, we can use numerical methods, as described in [25], to invert the Laplace transform of the scale function. We refer the reader to the papers [13], [17], and [19] for an updated account on explicit examples of scale functions and their properties.

In the sequel, for $a \in \mathbb{R}$, a function $f$, and a Borel measure $\mu$, we will use the notation $\int_{a}^{\infty} f(x) \mu(\mathrm{d} x)$ and $\int_{a+}^{\infty} f(x) \mu(\mathrm{d} x)$ to mean integration over the interval $[a, \infty)$ in the first case and integration over the interval $(a, \infty)$ in the second case. In particular, $\int_{a}^{\infty} f(x) \mu(\mathrm{d} x)=$ $f(a) \mu\{a\}+\int_{a+}^{\infty} f(x) \mu(\mathrm{d} x)$. We recall Bernstein's theorem (cf. [10, Chapter XIII.4]) which says that a real-valued function $f$ is completely monotone if and only if there exists a Borel measure $\mu$ such that $f(x)=\int_{0}^{\infty} \mathrm{e}^{-x t} \mu(\mathrm{d} t), x>0$. We now strengthen the conclusion of Theorem 3 in [20]. First we need the following proposition. 
Proposition 1. Suppose that $q>0$. Then

$$
\liminf _{x \rightarrow \infty} \mathrm{e}^{\Phi(q) x} W_{\Phi(q)}^{\prime}(x)=0 .
$$

Proof. Taking derivatives on both sides in (1) and using (2), we obtain

$$
\frac{\mathrm{d}}{\mathrm{d} x} \mathrm{E}_{x}\left(\exp \left(-q \tau_{0}^{-}\right) \mathbf{1}_{\left\{\tau_{0}^{-}<\infty\right\}}\right)=-\frac{q}{\Phi(q)} \mathrm{e}^{\Phi(q) x} W_{\Phi(q)}^{\prime}(x) .
$$

Suppose now that the conclusion of the proposition does not hold. Then $\mathrm{e}^{\Phi(q) x} W_{\Phi(q)}^{\prime}(x)$ will eventually be bounded from below by a strictly positive constant. It then follows that

$$
\lim _{x \rightarrow \infty} \mathrm{E}_{x}\left(\exp \left(-q \tau_{0}^{-}\right) \mathbf{1}_{\left\{\tau_{0}^{-}<\infty\right\}}\right)=-\infty,
$$

which contradicts the positivity of the expectation.

Theorem 2. Suppose that the Lévy measure $v$ has a completely monotone density and that $q>0$. Then the $q$-scale function can be written as

$$
W^{(q)}(x)=\frac{\mathrm{e}^{\Phi(q) x}}{\psi^{\prime}(\Phi(q))}-f(x), \quad x>0,
$$

where $f$ is a completely monotone function.

Proof. It was shown in [20] that if the Lévy measure $v$ has a completely monotone density then $W_{\Phi(q)}$ is a Bernstein function and, therefore, admits the representation

$$
W_{\Phi(q)}(x)=a+b x+\int_{0+}^{\infty}\left(1-\mathrm{e}^{-x t}\right) \xi(\mathrm{d} t), \quad x>0,
$$

where $a, b \geq 0$ and $\xi$ is a measure on $(0, \infty)$ satisfying $\int_{0+}^{\infty}(t \wedge 1) \xi(\mathrm{d} t)<\infty$. Since $q>0$, $W_{\Phi(q)}$ will be bounded and, therefore, $b=0$, and by using Fatou's lemma,

$$
\begin{aligned}
\xi(0, \infty) & =\int_{0+}^{\infty} \lim _{x \rightarrow \infty}\left(1-\mathrm{e}^{-x t}\right) \xi(\mathrm{d} t) \\
& \leq \lim _{x \rightarrow \infty} \int_{0+}^{\infty}\left(1-\mathrm{e}^{-x t}\right) \xi(\mathrm{d} t) \\
& =\lim _{x \rightarrow \infty} W_{\Phi(q)}(x)-a \\
& <\infty
\end{aligned}
$$

We now deduce from Proposition 1, (3), and Fatou's lemma that

$$
\begin{aligned}
0 & =\liminf _{x \rightarrow \infty} \mathrm{e}^{\Phi(q) x} W_{\Phi(q)}^{\prime}(x) \\
& =\liminf _{x \rightarrow \infty} \int_{0+}^{\infty} \mathrm{e}^{-x(t-\Phi(q))} t \xi(\mathrm{d} t) \\
& \geq \int_{0+}^{\infty} \liminf _{x \rightarrow \infty} \mathrm{e}^{-x(t-\Phi(q))} t \xi(\mathrm{d} t) \\
& \geq \Phi(q) \xi(0, \Phi(q)] .
\end{aligned}
$$


It follows that $\xi(0, \Phi(q)]=0$, and using (2) and (3), we can write

$$
\begin{aligned}
W^{(q)}(x) & =\mathrm{e}^{\Phi(q) x}(a+\xi(\Phi(q), \infty))-\int_{\Phi(q)+}^{\infty} \mathrm{e}^{-x(t-\Phi(q))} \xi(\mathrm{d} t) \\
& =\mathrm{e}^{\Phi(q) x}(a+\xi(\Phi(q), \infty))-\int_{0+}^{\infty} \mathrm{e}^{-x t} \xi(\mathrm{d} t+\Phi(q))
\end{aligned}
$$

Now the conclusion of the theorem follows by Bernstein's theorem and the fact that $a+$ $\xi(\Phi(q), \infty)=\lim _{x \rightarrow \infty} W_{\Phi(q)}(x)=1 / \psi^{\prime}(\Phi(q))$.

Denote by $W^{(q, n)}(x)$ the $n$th derivative of $W^{(q)}(x)$ for $x>0$ and $n=0,1,2, \ldots$

Corollary 1. Suppose that the Lévy measure v has a completely monotone density, that $q>0$, and that $n$ is an odd integer. Then $\log \left(W^{(q, n)}(x)\right)$ has a strictly positive second derivative for all $x>0$. Consequently, the function $W^{(q, n)}$ is strictly log-convex on $(0, \infty)$.

Proof. Suppose that the Lévy measure has a completely monotone density, that $q>0$, and that $n$ is an odd integer. Let

$$
f(x)=\frac{\mathrm{e}^{\Phi(q) x}}{\psi^{\prime}(\Phi(q))}-W^{(q)}(x) \text { and } \quad g(x)=-f^{\prime}(x) .
$$

By Theorem 2, $f$ and $g$ are completely monotone functions and

$$
W^{(q, n)}(x)=\frac{\Phi(q)^{n}}{\psi^{\prime}(\Phi(q))} \mathrm{e}^{\Phi(q) x}+g^{(n-1)}(x),
$$

where $g^{(n-1)}$ is the $(n-1)$ th derivative of $g$. Since $n$ is odd, $g^{(n-1)}$ is completely monotone and, hence, positive (in the weak sense). Furthermore, as $q>0, \Phi(q)>0$ and, therefore, $W^{(q, n)}(x)>0$ for $x>0$. This means that the following is well defined for $x>0$ :

$$
\begin{aligned}
h_{n}(x) & =\left(W^{(q, n)}(x)\right)^{2}\left[\log \left(W^{(q, n)}(x)\right)\right]^{\prime \prime} \\
& =W^{(q, n)}(x) W^{(q, n+2)}(x)-\left(W^{(q, n+1)}(x)\right)^{2} .
\end{aligned}
$$

We need to prove that $h_{n}(x)>0$ for all $x>0$. Using (5), we obtain

$$
\begin{aligned}
h_{n}(x)= & {\left[g^{(n-1)}(x) g^{(n+1)}(x)-\left(g^{(n)}(x)\right)^{2}\right] } \\
& +\frac{\Phi(q)^{n}}{\psi^{\prime}(\Phi(q))} \mathrm{e}^{\Phi(q) x}\left\{\Phi(q)^{2} g^{(n-1)}(x)+g^{(n+1)}(x)-2 \Phi(q) g^{(n)}(x)\right\} .
\end{aligned}
$$

By Bernstein's theorem and Hölder's inequality, for any completely monotone function $v$, we have, for some Borel measure $\mu$,

$$
v(x) v^{\prime \prime}(x)-\left(v^{\prime}(x)\right)^{2}=\int_{0}^{\infty} \mathrm{e}^{-x t} \mu(\mathrm{d} t) \int_{0}^{\infty} t^{2} \mathrm{e}^{-x t} \mu(\mathrm{d} t)-\left(\int_{0}^{\infty} t \mathrm{e}^{-x t} \mu(\mathrm{d} t)\right)^{2} \geq 0,
$$

and, therefore, $v$ is log-convex. Since $g^{(n-1)}$ is completely monotone, it is log-convex and, therefore, the expression between the square brackets in (6) is positive. Furthermore, the complete monotonicity of $g^{(n-1)}$ implies that each of the terms between the curly brackets in (6) is positive and, hence, $h_{n}(x) \geq 0$. As $\Phi(q)>0$, it suffices to prove that one of the terms between the curly brackets, say $g^{(n+1)}(x)$, is strictly positive. We do this by contradiction. 
Suppose that $g^{(n+1)}(x)=0$. Then it is easily seen from Bernstein's theorem that the function $f$ has to be equal to a constant. In this case, (4) implies that $f \equiv 0$. But this means that, for $\lambda>\Phi(q)$,

$$
\frac{1}{\psi(\lambda)-q}=\int_{0}^{\infty} \mathrm{e}^{-\lambda x} W^{(q)}(x) \mathrm{d} x=\int_{0}^{\infty} \frac{\mathrm{e}^{-(\lambda-\Phi(q)) x}}{\psi^{\prime}(\Phi(q))} \mathrm{d} x=\frac{1}{(\lambda-\Phi(q)) \psi^{\prime}(\Phi(q))} .
$$

Thus, $\psi(\lambda)$ is the Laplace exponent of a subordinator (consisting of just a single drift term). But subordinators were excluded from the definition of a spectrally negative Lévy process, which gives us the desired contradiction.

\section{Proof of Theorem 1}

In this section the proof of Theorem 1 will be given with the aid of a series of lemmas. The approach is similar to [3] and [20], namely, calculating the value of a barrier strategy where the barrier is arbitrary, then choosing the 'optimal' barrier, and finally putting this particular barrier strategy (or the take-the-money-and-run strategy) through a verification lemma.

First we recall what we mean by the term sufficiently smooth. A function $f: \mathbb{R} \rightarrow \mathbb{R}$ which vanishes on $(-\infty, 0)$ and which is right continuous at 0 is called sufficiently smooth at a point $x>0$ if $f$ is continuously differentiable at $x$ when $X$ is of bounded variation and is twice continuously differentiable at $x$ when $X$ is of unbounded variation. A function is then called sufficiently smooth if it is sufficiently smooth at all $x>0$. Note that we implicitly assume that a sufficiently smooth function is right continuous at 0 and that it equals 0 on $(-\infty, 0)$. We let $\Gamma$ be the operator acting on sufficiently smooth functions $f$, defined by

$$
\Gamma f(x)=\gamma f^{\prime}(x)+\frac{\sigma^{2}}{2} f^{\prime \prime}(x)+\int_{0+}^{\infty}\left(f(x-y)-f(x)+f^{\prime}(x) y \mathbf{1}_{\{0<y<1\}}\right) v(\mathrm{~d} y) .
$$

Lemma 1. (Verification lemma.) Suppose that $\hat{\pi}$ is an admissible dividend strategy such that $\left(v_{\hat{\pi}}-S\right)$ is sufficiently smooth, $v_{\hat{\pi}}(0) \geq S$, and, for all $x>0$,

$$
\max \left\{\Gamma v_{\hat{\pi}}(x)-q v_{\hat{\pi}}(x), 1-v_{\hat{\pi}}^{\prime}(x)\right\} \leq 0 .
$$

Then $v_{\hat{\pi}}(x)=v_{*}(x)$ for all $x \geq 0$ and, hence, $\hat{\pi}$ is an optimal strategy.

Proof. By the definition of $v_{*}$, it follows that $v_{\hat{\pi}}(x) \leq v_{*}(x)$ for all $x \geq 0$. We write $w:=v_{\hat{\pi}}$, and show that $w(x) \geq v_{\pi}(x)$ for all $\pi \in \Pi$ and all $x \geq 0$. First we suppose that $x>0$. We define, for $\pi \in \Pi$, the stopping time $\sigma_{0}^{\pi}$ by $\sigma_{0}^{\pi}=\inf \left\{t>0: U_{t}^{\pi} \leq 0\right\}$, and denote by $\Pi_{0}$ the following set of admissible dividend strategies:

$$
\Pi_{0}=\left\{\pi \in \Pi: \sigma_{0}^{\pi}=\sigma^{\pi} \mathrm{P}_{x} \text {-a.s. for all } x>0\right\} .
$$

Note that, when $X$ is of unbounded variation, $\Pi_{0}=\Pi$, but that $\Pi_{0}$ is a strictly smaller set than $\Pi$ when $X$ is of bounded variation. We claim that any $\pi \in \Pi$ can be approximated by dividend strategies from $\Pi_{0}$ in the sense that, for all $\varepsilon>0$, there exists $\pi_{\varepsilon} \in \Pi_{0}$ such that $v_{\pi}(x) \leq v_{\pi_{\varepsilon}}(x)+\varepsilon$ and, therefore, it is enough to show that $w(x) \geq v_{\pi}(x)$ for all $\pi \in \Pi_{0}$. Indeed, we can take $\pi_{\varepsilon}$ to be the strategy where you do not pay out any dividends until the stopping time $\kappa:=\inf \left\{t>0: L_{t}^{\pi} \geq \varepsilon\right\}$, then at that time point $\kappa$ pay out a dividend equal to the size of the overshoot of $L^{\pi}$ over $\varepsilon$ and afterwards follow the same strategy as $\pi$ until ruin occurs for the latter strategy, at which point you force ruin immediately. Note that $\pi_{\varepsilon} \in \Pi_{0}$ because 
if $\sigma_{0}^{\pi_{\varepsilon}}<\kappa$ then $\sigma_{0}^{\pi_{\varepsilon}}=\sigma^{\pi_{\varepsilon}}$ since until the first dividend payment is made, the process $U^{\pi_{\varepsilon}}$ is equal to $X$ and, for the spectrally negative Lévy process $X$, the first entry time in $(-\infty, 0]$ is equal a.s. to the first entry time in $(-\infty, 0)$, provided that $X_{0}>0$. Furthermore, if $\sigma_{0}^{\pi_{\varepsilon}} \geq \kappa$ and $\kappa<\infty$, then, since $U_{\kappa}^{\pi_{\varepsilon}}>U_{\kappa}^{\pi}$, we have $\sigma_{0}^{\pi_{\varepsilon}} \geq \sigma^{\pi}$ and, thus, $\sigma_{0}^{\pi_{\varepsilon}}=\sigma^{\pi_{\varepsilon}}$ since $\sigma^{\pi}=\sigma^{\pi_{\varepsilon}}$ on the event $\left\{\sigma_{0}^{\pi_{\varepsilon}} \geq \kappa, \kappa<\infty\right\}$ by construction.

We now assume without loss of generality that $\pi \in \Pi_{0}$, and we let $\widetilde{L}^{\pi}$ and $\widetilde{U}^{\pi}$ be the rightcontinuous modifications of $L^{\pi}$ and $U^{\pi}$. Note that since the filtration $\mathbb{F}$ was assumed to be right continuous, $\widetilde{L}^{\pi}$ and $\widetilde{U}^{\pi}$ are adapted processes. Let $\left(T_{n}\right)_{n \in \mathbb{N}}$ be the sequence of stopping times defined by $T_{n}=\inf \left\{t>0: \widetilde{U}_{t}^{\pi}>n\right.$ or $\left.\widetilde{U}_{t}^{\pi}<1 / n\right\}$. Since $\widetilde{U}^{\pi}$ is a semimartingale and $w$ is sufficiently smooth - in particular, $w$ and its derivatives are bounded on $[1 / n, n]$ for each $n$-we can use the change of variables/Itô's formula (cf. [21, Theorems II.31 and II.32]) on $\exp \left(-q\left(t \wedge T_{n}\right)\right) w\left(\widetilde{U}_{t \wedge T_{n}}^{\pi}\right)$ together with (7) to deduce that

$$
w\left(\tilde{U}_{0}^{\pi}\right) \geq \int_{0+}^{t \wedge T_{n}} \mathrm{e}^{-q s} \mathrm{~d} \widetilde{L}_{s}^{\pi}+\exp \left(-q\left(t \wedge T_{n}\right)\right) w\left(\tilde{U}_{t \wedge T_{n}}^{\pi}\right)+M_{t},
$$

where $\left\{M_{t}: t \geq 0\right\}$ is a zero-mean $\mathrm{P}_{x}$-martingale. The details yielding this inequality are given in Appendix A. Using the fact that $w \geq S$, which follows from the assumptions $w(0) \geq S$ and $w^{\prime} \geq 1$, taking expectations, letting $t$ and $n$ go to $\infty$, and using the monotone convergence theorem, we obtain

$$
w\left(\tilde{U}_{0}^{\pi}\right) \geq \mathrm{E}_{x}\left(\int_{0+}^{\sigma^{\pi}} \mathrm{e}^{-q s} \mathrm{~d} \tilde{L}_{s}^{\pi}\right)+S \mathrm{E}_{x}\left(\exp \left(-q \sigma^{\pi}\right)\right) .
$$

Note that we used here the fact that $T_{n} \nearrow \sigma^{\pi} \mathrm{P}_{x}$-a.s., which follows because $\pi \in \Pi_{0}$. Now, using the mean value theorem together with the assumption that $w^{\prime}(\cdot) \geq 1$ on $(0, \infty)$, we obtain

$$
w\left(\tilde{U}_{0}^{\pi}\right)=w\left(x-L_{0+}^{\pi}\right) \leq w(x)-L_{0+}^{\pi},
$$

and combining this with

$$
\mathrm{E}_{x}\left(\int_{0+}^{\sigma^{\pi}} \mathrm{e}^{-q s} \mathrm{~d} \widetilde{L}_{s}^{\pi}\right)=\mathrm{E}_{x}\left(\int_{0}^{\sigma^{\pi}} \mathrm{e}^{-q s} \mathrm{~d} L_{s}^{\pi}\right)-L_{0+}^{\pi}=v_{\pi}(x)-S \mathrm{E}_{x}\left(\exp \left(-q \sigma^{\pi}\right)\right)-L_{0+}^{\pi},
$$

we deduce that $w(x) \geq v_{\pi}(x)$ and, hence, we have proved $w(x) \geq v_{*}(x)$ for all $x>0$.

To complete the proof, note that $v_{*}$ is an increasing function (in the weak sense) since the set of admissible strategies is larger when the initial reserves are higher and, hence, because $w$ is right continuous at $0, v_{*}(0) \leq \lim _{x \downarrow 0} v_{*}(x) \leq \lim _{x \downarrow 0} w(x)=w(0)$.

Proposition 2. Assume that $W^{(q)}$ is continuously differentiable on $(0, \infty)$. The value function of the barrier strategy at level $a \geq 0$ is given by

$$
v_{a}(x)= \begin{cases}S Z^{(q)}(x)+W^{(q)}(x)\left(\frac{1-q S W^{(q)}(a)}{W^{(q)^{\prime}}(a)}\right) & \text { if } x \leq a, \\ x-a+S Z^{(q)}(a)+W^{(q)}(a)\left(\frac{1-q S W^{(q)}(a)}{W^{(q) \prime}(a)}\right) & \text { if } x>a .\end{cases}
$$

Proof. Clearly, the proposition only needs to be proved for $0 \leq x \leq a$. Let $x \in[0, a]$. By Avram et al. [3, Proposition 1], it follows that

$$
\mathrm{E}_{x}\left(\int_{0}^{\sigma^{a}} \mathrm{e}^{-q t} \mathrm{~d} L_{t}^{a}\right)=\frac{W^{(q)}(x)}{W^{(q)^{\prime}}(a)} .
$$


Since

$$
\sigma^{a}=\inf \left\{t>0: X_{t}-L_{t}^{a}<0\right\}=\inf \left\{t>0:\left(\sup _{0 \leq s<t} X_{s}\right) \vee a-X_{t}>a\right\},
$$

it follows from [2, Theorem 1] that

$$
\mathrm{E}_{x}\left(\exp \left(-q \sigma^{a}\right)\right)=Z^{(q)}(x)-W^{(q)}(x) \frac{q W^{(q)}(a)}{W^{(q)^{\prime}}(a)} .
$$

This completes the proof.

Define the function $\zeta:[0, \infty) \rightarrow \mathbb{R}$ by

$$
\zeta(x)=\frac{1-q S W^{(q)}(x)}{W^{(q) \prime}(x)} \quad \text { for } x>0
$$

and $\zeta(0)=\lim _{x \downarrow 0} \zeta(x)$. We now define the (candidate) optimal barrier level by

$$
a^{*}(S)=\sup \{a \geq 0: \zeta(a) \geq \zeta(x) \text { for all } x \geq 0\} .
$$

Hence, $a^{*}(S)$ is the last point where $\zeta$ attains its global maximum. Note that $a^{*}(0)$ is the point $a^{*}$ mentioned in Section 1. In the sequel we will write $a^{*}$ instead of $a^{*}(0)$.

Proposition 3. Suppose that $W^{(q)}$ is continuously differentiable on $(0, \infty)$. Then $a^{*}(S)<\infty$.

Proof. Define

$$
f(x)=\zeta(x)+\frac{q S}{\Phi(q)}=\frac{1+q S\left(\Phi(q)^{-1} W^{(q)^{\prime}}(x)-W^{(q)}(x)\right)}{W^{(q)^{\prime}}(x)} .
$$

Since $\lim _{x \rightarrow \infty} W^{(q)}(x) / W^{(q)^{\prime}}(x)=1 / \Phi(q)$ (see, e.g. [3, Section 3.3]) and $W^{(q)}$ is continuously differentiable, it follows that $\lim _{x \rightarrow \infty} f(x)=0$ and $f$ is continuous. Hence, $a^{*}(S)<\infty$ if there exists $x \geq 0$ such that $f(x)>0$. But, by (2),

$$
f(x)=\frac{1+(q S / \Phi(q)) \mathrm{e}^{\Phi(q) x} W_{\Phi(q)}^{\prime}(x)}{W^{(q)^{\prime}}(x)},
$$

and, thus, by Proposition 1, there exists $x \geq 0$ such that $f(x)>0$. This completes the proof.

Note that when $a^{*}(S)>0$ and $W^{(q)}$ is twice continuously differentiable, then $\zeta^{\prime}\left(a^{*}(S)\right)=0$. Furthermore, it is easily seen that if an optimal strategy is formed by a barrier strategy then the barrier strategy at $a^{*}(S)$ has to be an optimal strategy.

Lemma 2. Suppose that $W^{(q)}$ is sufficiently smooth and that

$$
\zeta(a) \geq \zeta(b) \text { for all } a, b \text { such that } a^{*}(S) \leq a \leq b .
$$

Then the following statements hold.

(i) If $\zeta\left(a^{*}(S)\right) \geq 0$ then the barrier strategy at $a^{*}(S)$ is an optimal strategy.

(ii) If $a^{*}(S)=0$ and $\zeta(0) \leq 0$, then the take-the-money-and-run strategy is optimal. 
Note that Lemma 2 is a generalization of Theorem 2 of [20]. Indeed, when $S=0, \zeta\left(a^{*}\right)=$ $1 / W^{(q) \prime}\left(a^{*}\right)>0$ and condition (9) transforms into the condition that $W^{(q)^{\prime}}$ is increasing on $\left(a^{*}, \infty\right)$.

Proof of Lemma 2. We first prove (i) by showing that $v_{a^{*}(S)}$ satisfies the conditions of the verification lemma. Using (9), all the conditions of the verification lemma can be proved following the same arguments as in the proofs of Lemma 5 and Theorem 2 of [20], with the exception being the condition that $v_{a^{*}(S)}(0) \geq S$. (Note that in deducing the analogue of Equation (4) of [20], we also use the fact that $\left(\exp \left(-q\left(t \wedge \tau_{0}^{-} \wedge \tau_{a}^{+}\right)\right) Z^{(q)}\left(X_{t \wedge \tau_{0}^{-} \wedge \tau_{a}^{+}}\right)\right)_{t \geq 0}$ is a $\mathrm{P}_{x}$-martingale; cf. [15, p. 229].) The missing condition now follows from

$$
v_{a^{*}(S)}(0)=S Z^{(q)}(0)+W^{(q)}(0) \zeta\left(a^{*}(S)\right) \geq S,
$$

where the inequality follows from the assumption that $\zeta\left(a^{*}(S)\right) \geq 0$.

For case (ii), we prove that $v_{\text {run }}$ satisfies the conditions of the verification lemma. Note that since $v_{\text {run }}(x)=x+S$ for $x \geq 0$, the only nontrivial thing to show is that $(\Gamma-q) v_{\text {run }}(x) \leq 0$ for all $x>0$. This can be achieved by mimicking the proof of Theorem 2 of [20], which involves proving that

$$
\lim _{y \uparrow x}(\Gamma-q)\left(v_{\text {run }}-v_{x}\right)(y) \leq 0 \text { for } x>0 .
$$

Note that in order to prove the above inequality, we use the fact that $v_{\text {run }}(0) \geq v_{x}(0)$, which follows from $\zeta(x) \leq 0$, and the latter is due to the assumption that $\zeta(0) \leq 0$ and $a^{*}(S)=0$ (combined with (9)).

Proof of Theorem 1. Since the case in which $S=0$ was proved in [20], we assume without loss of generality that $S \neq 0$. Note that, by Theorem $2, W^{(q)}$ is infinitely differentiable (this was proved for the first time in [6]) and, therefore, certainly smooth enough. Furthermore, note that $W^{(q) \prime \prime}$ is strictly negative on $\left(0, a^{*}\right)$, strictly positive on $\left(a^{*}, \infty\right)$, and if $a^{*}>0$ then $W^{(q) \prime \prime}\left(a^{*}\right)=0$. We will show that

$$
\zeta \text { is strictly increasing on }\left(0, a^{*}(S)\right) \text { and strictly decreasing on }\left(a^{*}(S), \infty\right),
$$

from which it follows that $a^{*}(S)$ is the only point where $\zeta$ has a local/global maximum and that (9) holds.

First note that with $g(x)=-q S W^{(q)^{\prime}}(x) / W^{(q) \prime \prime}(x)$ for $x \in(0, \infty) \backslash\left\{a^{*}\right\}$, the following differential equation holds for $\zeta$ :

$$
\zeta^{\prime}(x)=-\frac{W^{(q) \prime \prime}(x)}{W^{(q)^{\prime}}(x)}(\zeta(x)-g(x)), \quad x \in(0, \infty) \backslash\left\{a^{*}\right\} .
$$

From this, it follows that, for $x \in\left(0, a^{*}\right)$,

$$
\begin{array}{lll}
\zeta^{\prime}(x)>0 & \text { if and only if } & \zeta(x)>g(x), \\
\zeta^{\prime}(x)<0 & \text { if and only if } & \zeta(x)<g(x), \\
\zeta^{\prime}(x)=0 & \text { if and only if } & \zeta(x)=g(x),
\end{array}
$$

and, for $x \in\left(a^{*}, \infty\right)$,

$$
\begin{array}{lll}
\zeta^{\prime}(x)>0 & \text { if and only if } & \zeta(x)<g(x), \\
\zeta^{\prime}(x)<0 & \text { if and only if } & \zeta(x)>g(x), \\
\zeta^{\prime}(x)=0 & \text { if and only if } & \zeta(x)=g(x) .
\end{array}
$$


Suppose that $S>0$. Since

$$
\zeta^{\prime}(x)=\frac{q S\left[W^{(q)}(x) W^{(q) \prime \prime}(x)-\left(W^{(q) \prime}(x)\right)^{2}\right]-W^{(q) \prime \prime}(x)}{\left(W^{(q) \prime}(x)\right)^{2}},
$$

where the expression between the square brackets is negative due to the log-concavity of $W^{(q)}$, it follows that $\zeta^{\prime}(x)<0$ on $\left(a^{*}, \infty\right)$ and, therefore, $a^{*}(S) \leq a^{*}$. If $a^{*}=0,(10)$ now holds, so we can assume without loss of generality that $a^{*}>0$. Then $\lim _{x \uparrow a^{*}} g(x)=\infty$ and (11)(13) imply that $a^{*}(S) \neq a^{*}$ and, thus, $a^{*}(S)<a^{*}$. By the strict log-convexity of $W^{(q) \prime}$ (Corollary 1$), g$ is strictly increasing on $\left(0, a^{*}\right)$. The foregoing and (11)-(13) then imply that either $\zeta$ intersects $g$ exactly once on $(0, \infty)\left(\right.$ at $\left.a^{*}(S)\right)$ and (10) holds or that $\zeta^{\prime}(x)<0$ for all $x>0$, and in that case $a^{*}(S)=0$. Hence, (10) holds when $S>0$.

Suppose now that $S<0$ and $a^{*}>0$. Then $\zeta$ is strictly positive on $(0, \infty)$ by definition and $g$ is strictly negative on $\left(0, a^{*}\right)$. Hence, $a^{*}(S) \geq a^{*}$. Due to the strict log-convexity of $W^{(q) \prime}$, $g$ is in this case strictly decreasing on $\left(a^{*}, \infty\right)$ and combined with (14)-(16) and the fact that $\lim _{x \downarrow a^{*}} g(x)=\infty$, this implies that $\zeta$ and $g$ intersect each other exactly once, $a^{*}(S)>a^{*}$, and that (10) holds.

This leaves the final case when $S<0$ and $a^{*}=0$. If $\zeta(0) \geq g(0)$ then (14)-(16) and $g$ being strictly decreasing on $(0, \infty)$ imply that $\zeta$ is strictly decreasing on $(0, \infty)$ and, hence, $a^{*}(S)=0$. If $\zeta(0)<g(0)$ then $a^{*}(S)>0$ and, furthermore, (10) holds by the same arguments as before.

Suppose now that $\sigma>0$, or $v(0, \infty)=\infty$, or $v(0, \infty)<\infty$ and $S \leq c / q$. Then from the values of $W^{(q)}(0)$ and $W^{(q) \prime}(0)$ given in Section 2 , it follows that $\zeta(0) \geq 0$ and, hence, $\zeta\left(a^{*}(S)\right) \geq 0$ by the definition of $a^{*}(S)$. Thus, part (i) of the theorem follows from Lemma 2(i).

To prove part (ii), suppose that $\sigma=0, v(0, \infty)<\infty$, and $S>c / q$. This implies that $S>0$ and $\zeta(0)<0$. If $a^{*}=0$ then $a^{*}(S)=0$ since $S>0$. If $a^{*}>0$ then $g(0)>0$ and, hence, by (11)-(12) and (17), $\zeta^{\prime}(x)<0$ for all $x>0$ and, therefore, $a^{*}(S)=0$. Part (ii) now follows from Lemma 2(ii).

\section{Appendix A}

We give here the details which lead to (8).

Applying the change of variables/Itô's formula to $\exp \left(-q\left(t \wedge T_{n}\right)\right) w\left(\widetilde{U}_{t \wedge T_{n}}^{\pi}\right)$ gives

$$
\begin{aligned}
\exp \left(-q\left(t \wedge T_{n}\right)\right) w\left(\widetilde{U}_{t \wedge T_{n}}^{\pi}\right)-w\left(\widetilde{U}_{0}^{\pi}\right)= & \int_{0+}^{t \wedge T_{n}} \mathrm{e}^{-q s}\left(\frac{\sigma^{2}}{2} w^{\prime \prime}\left(\widetilde{U}_{s-}^{\pi}\right)-q w\left(\widetilde{U}_{s-}^{\pi}\right)\right) \mathrm{d} s \\
& +\int_{0+}^{t \wedge T_{n}} \mathrm{e}^{-q s} w^{\prime}\left(\widetilde{U}_{s-}^{\pi}\right) \mathrm{d}\left(X_{s}-\left(\widetilde{L}_{s}^{\pi}\right)^{c}\right) \\
& +\sum_{0<s \leq t \wedge T_{n}} \mathrm{e}^{-q s}\left(\Delta w\left(\widetilde{U}_{s}^{\pi}\right)-w^{\prime}\left(\widetilde{U}_{s-}^{\pi}\right) \Delta X_{s}\right),
\end{aligned}
$$

where we used the following notation: $\Delta \widetilde{U}_{s}^{\pi}=\widetilde{U}_{s}^{\pi}-\widetilde{U}_{s-}^{\pi}, \Delta w\left(\tilde{U}_{s}^{\pi}\right)=w\left(\widetilde{U}_{s}^{\pi}\right)-w\left(\tilde{U}_{s-}^{\pi}\right)$, and $\left(\widetilde{L}_{s}^{\pi}\right)^{c}=\widetilde{L}_{s}^{\pi}-\sum_{0<u \leq s} \Delta \widetilde{L}_{u}^{\pi}$. Note that we have used here the fact that the continuous 
martingale part of $X$ is its Gaussian part. We can easily verify that

$$
\begin{aligned}
& \sum_{0<s \leq t \wedge T_{n}} \mathrm{e}^{-q s}\left(\Delta w\left(\tilde{U}_{s}^{\pi}\right)-w^{\prime}\left(\tilde{U}_{s-}^{\pi}\right) \Delta X_{s}\right) \\
& =\sum_{0<s \leq t \wedge T_{n}} \mathrm{e}^{-q s}\left(\Delta w\left(\widetilde{U}_{s-}^{\pi}+\Delta X_{s}\right)-w^{\prime}\left(\widetilde{U}_{s-}^{\pi}\right) \Delta X_{s}\right) \\
& \quad-\sum_{0<s \leq t \wedge T_{n}} \mathrm{e}^{-q s}\left(w\left(X_{s}-\widetilde{L}_{s-}^{\pi}\right)-w\left(X_{s}-\widetilde{L}_{s-}^{\pi}-\Delta \widetilde{L}_{s}^{\pi}\right)\right) .
\end{aligned}
$$

Since, by the admissibility of $L^{\pi}$, we have $\Delta \widetilde{L}_{s}^{\pi} \leq X_{s}-\widetilde{L}_{s-}^{\pi}$, and so by the mean value theorem and the hypothesis, $w^{\prime} \geq 1$,

$$
w\left(X_{s}-\widetilde{L}_{s-}^{\pi}\right)-w\left(X_{s}-\widetilde{L}_{s-}^{\pi}-\Delta \widetilde{L}_{s}^{\pi}\right) \geq \Delta \widetilde{L}_{s}^{\pi} \quad \text { for } 0<s<t \wedge T_{n} .
$$

Combining (18), (19), and (20) leads to

$$
\begin{aligned}
& \mathrm{e}^{-q\left(t \wedge T_{n}\right)} w\left(\tilde{U}_{t \wedge T_{n}}^{\pi}\right)-w\left(\widetilde{U}_{0}^{\pi}\right) \\
& \leq \int_{0+}^{t \wedge T_{n}} \mathrm{e}^{-q s}\left(\frac{\sigma^{2}}{2} w^{\prime \prime}\left(\tilde{U}_{s-}^{\pi}\right)-q w\left(\tilde{U}_{s-}^{\pi}\right)\right) \mathrm{d} s \\
& +\int_{0+}^{t \wedge T_{n}} \mathrm{e}^{-q s} w^{\prime}\left(\tilde{U}_{s-}^{\pi}\right) \mathrm{d}\left(X_{s}-\left(\tilde{L}_{s}^{\pi}\right)^{c}\right) \\
& +\sum_{0<s \leq t \wedge T_{n}} \mathrm{e}^{-q s}\left(\Delta w\left(\tilde{U}_{s-}^{\pi}+\Delta X_{s}\right)-w^{\prime}\left(\tilde{U}_{s-}^{\pi}\right) \Delta X_{s}-\Delta \widetilde{L}_{s}^{\pi}\right) \\
& =\int_{0+}^{t \wedge T_{n}} \mathrm{e}^{-q s}(\Gamma-q) w\left(\tilde{U}_{s-}^{\pi}\right) \mathrm{d} s-\int_{0+}^{t \wedge T_{n}} \mathrm{e}^{-q s} w^{\prime}\left(\tilde{U}_{s-}^{\pi}\right) \mathrm{d}\left(\tilde{L}_{s}^{\pi}\right)^{c}-\sum_{0<s \leq t \wedge T_{n}} \mathrm{e}^{-q s} \Delta \widetilde{L}_{s}^{\pi} \\
& +\left\{\int_{0+}^{t \wedge T_{n}} \mathrm{e}^{-q s} w^{\prime}\left(\widetilde{U}_{s-}^{\pi}\right) \mathrm{d}\left(X_{s}-\gamma s-\sum_{0<u \leq s} \Delta X_{u} \mathbf{1}_{\left\{\left|\Delta X_{u}\right| \geq 1\right\}}\right)\right\} \\
& +\left\{\sum_{0<s \leq t \wedge T_{n}} \mathrm{e}^{-q s}\left(\Delta w\left(\widetilde{U}_{s-}^{\pi}+\Delta X_{s}\right)-w^{\prime}\left(\widetilde{U}_{s-}^{\pi}\right) \Delta X_{s} \mathbf{1}_{\left\{\left|\Delta X_{s}\right|<1\right\}}\right)\right. \\
& \left.-\int_{0+}^{t \wedge T_{n}} \int_{0+}^{\infty} \mathrm{e}^{-q s}\left(w\left(\tilde{U}_{s-}^{\pi}-y\right)-w\left(\tilde{U}_{s-}^{\pi}\right)+w^{\prime}\left(\tilde{U}_{s-}^{\pi}\right) y \mathbf{1}_{\{0<y<1\}}\right) v(\mathrm{~d} y) \mathrm{d} s\right\} .
\end{aligned}
$$

By the Lévy-Itô decomposition, the expression between the first pair of curly brackets is a zeromean martingale and, by the compensation formula (cf. [15, Corollary 4.6]), the expression between the second pair of curly brackets is also a zero-mean martingale. Now using (7), inequality (8) follows.

\section{References}

[1] Albrecher, H., Renaud, J.-F. and Zhou, X. (2008). A Lévy insurance risk process with tax. J. Appl. Prob. 45, 363-375.

[2] Avram, F., Kyprianou, A. E. and Pistorius, M. R. (2004). Exit problems for spectrally negative Lévy processes and applications to (Canadized) Russian options. Ann. Appl. Prob. 14, 215-235.

[3] Avram, F., Palmowski, Z. and Pistorius, M. R. (2007). On the optimal dividend problem for a spectrally negative Lévy process. Ann. Appl. Prob. 17, 156-180. 
[4] Azcue, P. and Muler, N. (2005). Optimal reinsurance and dividend distribution policies in the CramérLundberg model. Math. Finance 15, 261-308.

[5] Boguslavskaya, E. V. (2008). Optimization problems in financial mathematics: explicit solutions for diffusion models. Doctoral Thesis, University of Amsterdam.

[6] Chan, T. and Kyprianou, A. E. (2007). Smoothness of scale functions for spectrally negative Lévy processes. Preprint.

[7] De Finetti, B. (1957). Su un'impostazion alternativa della teoria collecttiva del rischio. Trans. XVth Internat. Congress Actuaries 2, 433-443.

[8] Dufresne, F. And Gerber, H. U. (1993). The probability of ruin for the inverse Gaussian and related processes. Insurance Math. Econom. 12, 9-22.

[9] Dufresne, F., Gerber, H. U. And Shiu, E. S. W. (1991). Risk theory with the gamma process. Astin Bull. 21, 177-192.

[10] Feller, W. (1971). An Introduction to Probability Theory and Its Applications, Vol. 2, 2nd edn. John Wiley, New York.

[11] Furrer, H. (1998). Risk processes perturbed by $\alpha$-stable Lévy motion. Scand. Acturial J. 1998, 59-74.

[12] Gerber, H. U. (1969). Entscheidungskriterien für den zusammengesetzten Poisson-Prozess. Mit. Verein. Schweiz. Versicherungsmath. 69, 185-227.

[13] Hubalek, F. and Kyprianou, A. E. (2007). Old and new examples of scale functions for spectrally negative Lévy processes. Preprint. Available at http://arxiv.org/abs/0801.0393v1.

[14] Huzak, M., Perman, M., ŠIKić, H. AND VondračEK, Z. (2004). Ruin probabilities and decompositions for general perturbed risk processes. Ann. Appl. Prob. 14, 1378-1397.

[15] Kyprianou, A. E. (2006). Introductory Lectures on Fluctuations of Lévy Processes with Applications. Springer, Berlin.

[16] Kyprianou, A. E. and Palmowski, Z. (2007). Distributional study of de Finetti's dividend problem for a general Lévy insurance risk process. J. Appl. Prob. 44, 428-443.

[17] Kyprianou, A. E. AND Rivero, V. (2008). Special, conjugate and complete scale functions for spectrally negative Lévy processes. Electron. J. Prob. 13, 1672-1701.

[18] Kyprianou, A. E. And Surya, B. A. (2007). Principles of smooth and continuous fit in the determination of endogenous bankruptcy levels. Finance Stoch. 11, 131-152.

[19] Kyprianou, A. E., Rivero, V. and Song, R. (2008). Convexitity and smoothness of scale functions and de Finetti's control problem. Preprint. Available at http://arXiv.org/abs/0801.1951v2.

[20] Loeffen, R. L. (2008). On optimality of the barrier strategy in de Finetti's dividend problem for spectrally negative Lévy processes. Ann. Appl. Prob. 18, 1669-1680.

[21] Protter, P. (2005). Stochastic Integration and Differential Equations, 2nd edn. Springer, Berlin.

[22] RADNER, R. AND SHEPP, L. (1996). Risk vs. profit potential: a model for corporate strategy. J. Econom. Dynamics Control 20, 1373-1393.

[23] Renaud, J. F. and Zhou, X. (2007). Distribution of the present value of dividend payments in a Lévy risk model. J. Appl. Prob. 44, 420-427.

[24] Shreve, S. E., Lehoczky, J. P. And Gaver, D. P. (1984). Optimal consumption for general diffusions with absorbing and reflecting barriers. SIAM J. Control Optimization 22, 55-75.

[25] Surya, B. A. (2008). Evaluating scale functions of spectrally negative Lévy processes. J. Appl. Prob. 45, 135-149.

[26] Thonhauser, S. And Albrecher, H. (2007). Dividend maximization under consideration of the time value of ruin. Insurance Math. Econom. 41, 163-184.

[27] Van Tiel, J. (1984). Convex Analysis. John Wiley, New York. 